\title{
SQUAMOUS CELL CARCINOMA OF THE CAUDAL NASAL SEPTUM AND THE NASAL VESTIBULE: A CASE REPORT
}

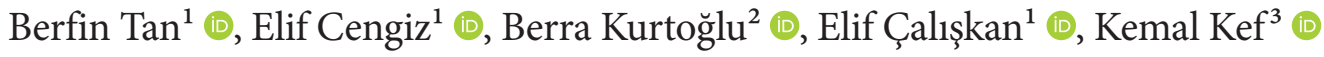

${ }^{1}$ Trakya University School of Medicine, Edirne, TURKEY

${ }^{2}$ Gazi University School of Medicine, Ankara, TURKEY

${ }^{3}$ Department of Otolaryngology, Private Keşan Hospital, Edirne, TURKEY

\begin{abstract}
Aims: The nasal septum is an unusual place for malignant tumors to occur. The condition may be accompanied by non-specific symptoms that may cause delays in seeking medical care or may misguide the physician due to the condition's rarity. We hereby aim to present a rare case of a squamous cell carcinoma in a 65-year-old male patient with recently progressing symptoms. Case Report: A 65-year-old male patient was admitted to Private Keşan Hospital's EarNose-Throat Department with swelling and wounds in the nose, and inability to breathe complaints. After the biopsy, pathological examination revealed moderately differentiated squamous cell carcinoma. The patient was operated with success; the mass was thoroughly dissected and a full-thickness skin graft from the left supraclavicular region was used in the reconstruction of defected areas. Recovery was uneventful due to early diagnosis and admission, with no relapse or cosmetic concern during monthly visits in the following years. Conclusion: As seen in this case, full-thickness skin grafts can be successfully used in intranasal and extranasal reconstructions, provided that they are obtained from areas with appropriate thickness. Early diagnosis and frequent monitoring are crucial in patients with squamous cell carcinoma since these tumors respond exceptionally well to treatment; although the recurrence rate is remarkably high. Keywords: Nasal septum, squamous cell carcinoma, grafts
\end{abstract}

\section{INTRODUCTION}

Tumors of the nasal cavity and paranasal sinuses make up less than $3 \%$ of upper aerodigestive tract malignancies and less than $1 \%$ of all malignant tumors (1). Malignancies of the nasal septum are rare: only $9 \%$ of sinonasal malignancies are primary nasal septum malignancies (2). Even though their association with nasal septum malignancies is not yet fully known (partially because of the absence of standardized classification of nasal septum malignancies), smoking tobacco or occupational exposure to wood dust, petroleum products, chemicals used in nickel refining, leatherworking, textile, and isopropyl alcohol manufactory are among the risk factors for sinonasal adenocarcinomas (3-6).

Nasal septum tumors are generally seen in the 4 th and 5 th decades of males' lives (7). There is an increased risk of nasal carcinoma in tobacco smokers (8). Squamous cell carcinoma (SCC) is the most common malignant tumor of the nasal septum, which accounts for $66 \%$ of primary carcinomas of the nasal septum $(6,9)$.

Patients with SCC of the nasal septum may delay seeking medical care or their diagnosis may be delayed due to the non-specific symptoms that are similar to everyday rhinologic complaints. $\mathrm{Pa}-$ tients present symptoms such as nasal obstruction, a nasal mass, recurrent epistaxis, pain, rhinorrhea, and facial edema. Common physical examination findings are nasal deformity, polyp, mucosal ulceration, and septal perforation (5, 9-11). Tissue biopsy is used for the diagnosis of nasal septum tumors (5).

There are various treatment approaches for nasal defects. Skin flaps from the areas adjacent to the nose such as cheeks and nasolabial sulcus can be used to repair the area (12). Median or paramedian forehead flaps can also be used (13). A full layer of skin cartilage graft can be taken from the auricle, especially in alar wing repairs that require skin and cartilage association (14). Repairment of the defected areas can be done with silicon septal buttons for defects that occur only in the septum, if there is no suitable tissue to cover the defected area, or if the patient especially requests it $(15,16)$.

In this article, we present the treatment approach to a rare case of SCC of the nasal septum.

\section{CASE REPORT}

A 65-year-old male patient was admitted to the Ear-NoseThroat Department of Private Keşan Hospital with swelling and wounds in the nose, as well as inability to breathe. The patient had been a tobacco smoker for 30 years, smoking one pack a day, and had quit smoking 5 years ago. The patient stated that he had these complaints for 5-6 months, yet they progressed recently.

The patient went under ENT examination. The right nasal vestibule and cavity were observed as normal in the nasal examination but the left nasal cavity was filled with ulcerovegetant mass. A biopsy was recommended because of the patient's history and the appearance of the mass. The biopsy was performed under local anesthesia. Pathological examination revealed moderately differentiated SCC. The patient's head and neck examination, ultrasonography, and laboratory tests showed no pathological lymphadenopathy. 
Detailed information about his disease and the pathological result was given to the patient. The patient was told that surgery was required to remove the mass in his nose and how the surgery would be performed. An informed consent form was obtained from the patient. To not disrupt the blood flow to the nasal cavity, surgery under general anesthesia was decided, resulting in a successful functional reconstruction due to the size and diffuseness of the tumor. After the patient's tests and consultations were evaluated as suitable for anesthesia, the day of surgery was determined.

The operation was started as the patient went under general anesthesia, in the supine position with his head turned slightly to his right. The left nasal cavity was made visible after making a left lateral rhinotomy incision. It was observed that the tumor involved the left anterior part of the septum, the skin of the left nasal vestibule, and the left nasal ala (Figure 1). The incision, which had an adequate safety area from the tumor, was started from the left nasal vestibule.

When the septum was reached, the opposite mucosa was partly preserved, the mass was removed with the cartilage and dissected until the safe area behind was reached. Starting from the intact skin edge inside the ala, on the upper side, the tumor tissue was totally removed and continued downwards with sharp dissection. The tumor was approximately $15 \times 20 \times 10 \mathrm{~mm}$ in size. Surgical margins were marked with different suture materials. Where these margins coincided were written in detail before sending the samples to the pathology laboratory. Intranasal surgical margins were re-evaluated. The bleeding was controlled.

A left supraclavicular full-thickness skin graft was taken for the repairment of the septal, vestibular, and alar defect. The skin graft was $2 \times 3 \mathrm{~cm}$ in size, the same as the defected area. The graft was sutured with an absorbable suture material inside the nose to the skin of the vestibule in the front, ala on the top and side, and septal mucosa on the back (Figure 2). The graft tissue was supported by placing an absorbable hemostatic gelatin sponge in the nasal cavity. There were no preoperative or postoperative complications.

No problem with the nutrition of the graft was observed. The patient was taken to his bed in good condition. Following the graft healing, no intranasal or extranasal problems were observed. The nasal passage continued to be completely open. There was no cosmetic problem in the nasal appearance (Figure 3).

Histopathological examination of the removed tissue confirmed moderately differentiated SCC. All of the surgical margins marked were reported as tumor-free and safe. The supraclavicular area (donor area) where the skin graft was taken, also healed without any problem.

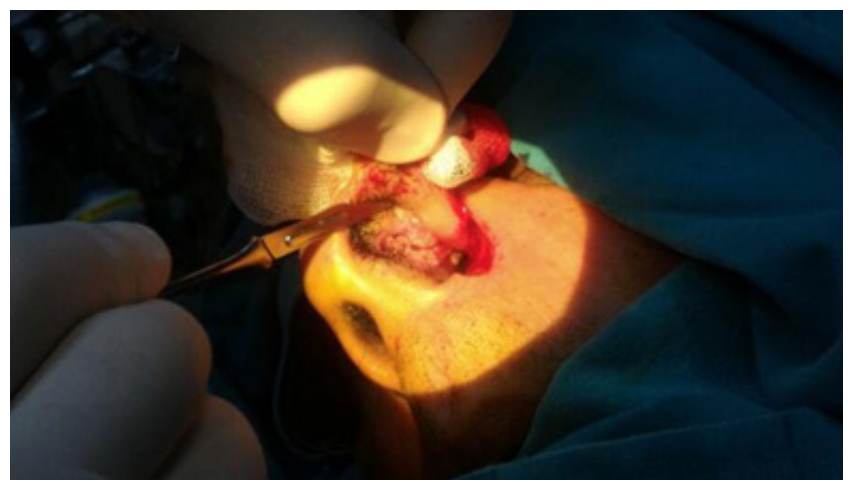

Figure 1: Intraoperative image of the tumor. Tumor involves the left anterior part of the septum, the skin of the left nasal vestibule, and the left nasal ala.
Following the discharge of the patient, a detailed epicrisis was written and sent to the oncology clinic, with the pathology reports attached. As a result of the patient's oncological examination, it was stated that the surgery performed was sufficient, and did not require any additional treatment or procedure.

The patient was called for monthly follow-up visits during the first year after the operation and was followed up for both local relapse and metastasis. Local areas and the areas with high drainage possibility were followed by ultrasonography in terms of lymphadenopathy. In the second year, he was called and followed up every three months. The patient completed his third year without local relapse and metastasis. During all these follow-ups, the patient had no clinical complaints.

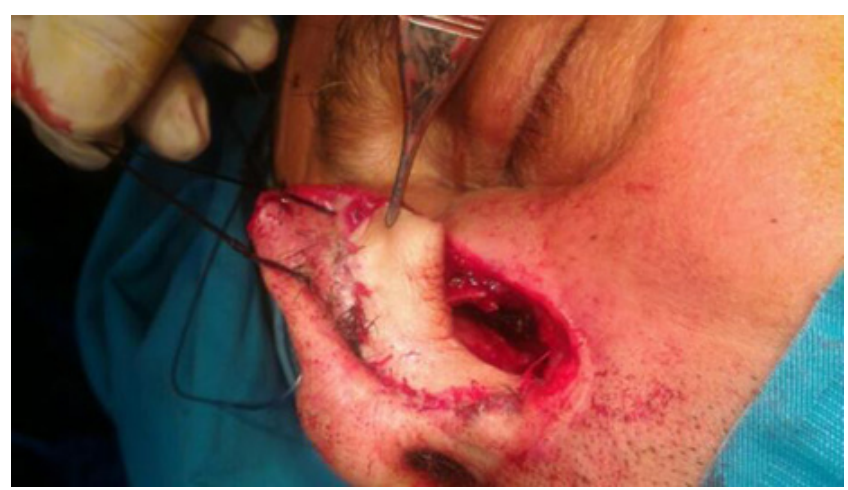

Figure 2: Placement of the supraclavicular skin graft to the nose.

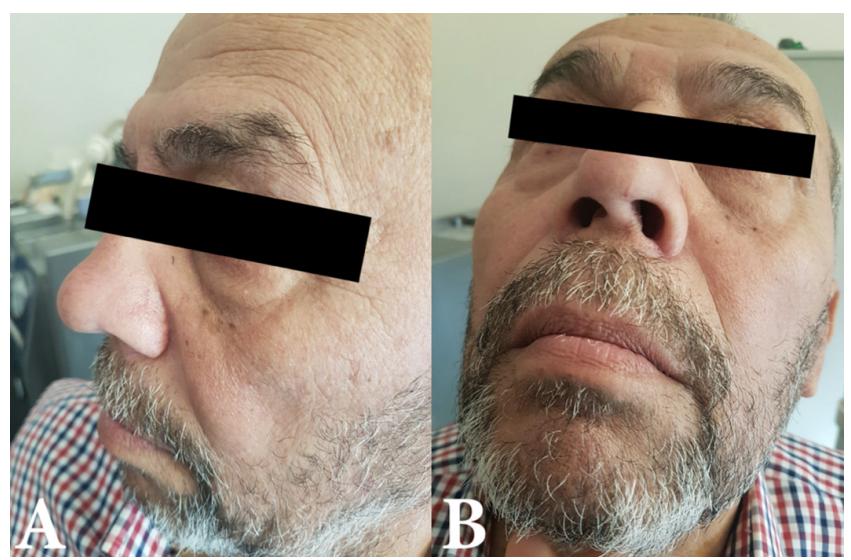

Figure 3: A, B: Patient's postoperative appearance.

\section{DISCUSSION}

To the best of our knowledge, fewer than 400 nasal septum malignancies had been reported up until the year 2010, making it quite a rare case (5). The diagnosis of nasal septum malignancies is often delayed due to the nonspecific symptoms. Tissue biopsy is the most straightforward way for diagnosis (5). Our patient came with nonspecific symptoms such as swelling and wounds in the nose, and an inability to breathe. With further examination an ulcerovegetant mass was observed in the left nasal cavity, then it was decided for the patient to have a biopsy. The final diagnosis after the pathological examination was moderately differentiated SCC. Correct surgical excision is the gold standard for the diagnosis and treatment of SCC (17). Our patient had surgery under general anesthesia and the tumor was removed successfully. 
After the surgery, septal, vestibular, and alar defects were seen. Various treatments can be applied to nasal defects. The case's specificities, patient's age, and medical history, the experience of the surgeon are the criteria in determining the appropriate way of reconstruction. Flaps of the cheek, nasolabial sulcus, median, and paramedian forehead or grafts obtained from conchal cartilage can be used for the repairment of nasal septal defects (12-14). Small or medium-sized defects can also be repaired with the advancement flaps, which can be applied in all anatomic regions of the nose (18). Silicon septal buttons can be used if there is no suitable tissue to cover the affected area $(15,16)$. In total or subtotal nasal reconstructions, forehead flaps may be considered primarily. However, since the face is the most striking part in terms of cosmetics, choosing a donor site further away from the face, and which will not cause cosmetic problems, will increase patient satisfaction. Refined aesthetic outcomes and preserving nasal functions are crucial in a nasal operation, thus the surgeon should decide the best and most suitable method for the patient. Sapthavee et al. (19) stated that skin grafts provided aesthetic outcomes comparable to those of local flap procedures. In this case, since the mucosal and cartilage tissue defects that occurred after the removal of the tumor were large, a large and solid tissue was required for repairment. Therefore, a full-thickness skin graft from the supraclavicular area was successfully used for the reconstruction of the defected areas.

When the diagnosis is made in the early stage, tumors respond well to treatment. Close and frequent monitoring after treatment is crucial in nasal septum malignancies because of its high local recurrence rate (7). Our patient made monthly follow-up visits during the first year after the operation and visited every three months in the second year. No local relapse, metastasis, or clinical complaints were observed for three years. This good prognosis and healing process of the patient may be a result of early diagnosis.

Our patient was a former smoker for 30 years and smoked around 1 cigarette pack per day. This is a risk factor for cancers, including nasal carcinomas. Bosch et al. (20) reviewed 40 patients with nasal cancer over 15 years. There was an association between patients who smoked from an early age (median age $=14$ years) and nasal cancer. Another study also found out that the highest risk of nasopharyngeal cancer was observed among men who have smoked for 30 years or more (21). Direct contact of the tobacco smoke on nasal mucosa may explain the increased incidence.

Due to the rarity of nasal septum malignancies, the understanding of the disease is still limited. We believe that this case report will provide physicians a different perspective and make a contribution to the literature.

In conclusion, full-thickness skin grafts can be successfully used in intranasal and extranasal reconstructions as long as they are obtained from the areas with appropriate thickness. Early diagnosis and frequent monitoring are crucial in those patients with squamous cell carcinoma, as these tumors respond exceptionally well to treatment but the recurrence rate is remarkably high.
Ethics Committee Approval: N/A

Informed Consent: Verbal informed consent was obtained from the patient. Conflict of Interest: The authors declared no conflict of interest

Author Contributions: Concept: BT, EC, BK, EÇ, KK. Design: BT, EC, BK, EÇ, KK. Supervision: BT, EC, BK, EÇ, KK. Resources: BT, EC, BK, EÇ, KK. Materials: BT, EC, BK, EÇ, KK. Data collection and/or Processing: BT, EC, BK, EÇ, KK. Analysis and/or Interpretation: BT, EC, BK, EÇ, KK. Literature Search: BT, EC, BK, EÇ, KK. Writing Manuscript: BT, EC, BK, EÇ, KK. Critical Review: BT, EC, BK, EÇ, KK.

Financial disclosure: The authors declared that this study received no financial support.

Editor-in-chief's Note: Four of the authors of this article, Berfin Tan, Elif Cengiz, Berra Kurtoğlu, Elif Çalışkan are members of the editorial board of Turkish Medical Student Journal. However, they did not take place at any stage of the editorial decision of the manuscript. The editors who evaluated this manuscript are from other institutions.

\section{REFERENCES}

1. Turner JH, Reh DD. Incidence and survival in patients with sinonasal cancer: A historical analysis of population-based data. Head Neck 2012;34(6):877-85.

2. Echeverria-Zumarraga M, Kaiser C, Gavilani C. Nasal septal carcinoma: Initial symptom of nasal septal perforation. J Laryngol Otol 1988;102(9):834-5.

3. Fornelli RA, Fedok FG, Wilson EP et al. Squamous cell carcinoma of the anterior nasal cavity: A dual institution review. Otolaryngol Head Neck Surg 2000;123(3):207-10.

4. Allen MW, Schwartz DL, Rana V et al. Long-term radiotherapy outcomes for nasal cavity and septal cancers. Int J Radiat Oncol Biol Phys 2008;71(2):401-6.

5. Ho YM, Coman WB. Nasal septum malignancy. ANZ J Surg 2011;81(7-8):533-6.

6. Cukurova I, Gümüsssoy M, Kaptaner S et al. Squamous cell carcinoma originating from the nasal septal perforation: A rare nasal tumor. Kulak Burun Bogaz Ihtis Derg 2014;24(3):177-80.

7. Gur OE, Ensari N, Senen D et al. Squamous cell carcinoma of nasal septum: Case report. J Ann Eu Med 2017;5(1):27-9

8. Beatty CW, Pearson BW, Kern EB. Carcinoma of the nasal septum: Experience with 85 cases. Otolaryngol Head Neck Surg 1982;90(1):90-4.

9. DiLeo MD, Miller RH, Rice JC et al. Nasal septal squamous cell carcinoma: A chart review and meta-analysis. Laryngoscope 1996;106(10):1218-22.

10. Weimert TA, Batsakis JG, Rice DH. Carcinomas of the nasal septum. J Laryngol Otol 1978;92:209-13.

11. LeLiever WC, Bailey BJ, Griffths C. Carcinoma of the nasal septum. Arch Otolaryngol 1984;110:748-51.

12. Ohlsén L. Closure of nasal septal perforation with a cutaneous flap and a perichondrocutaneous graft. Ann Plast Surg 1988;21(3):276-88.

13. Kheradmand AA, Garajei A, Motamedi MH. Nasal reconstruction: Experience using tissue expansion and forehead flap. J Oral Maxillofac Surg 2011;69(5):147884 .

14. Austin GK, Shockley WW. Reconstruction of nasal defects: Contemporary approaches. Curr Opin Otolaryngol Head Neck Surg 2016;24(5):453-60.

15. Osma U, Cüreoğlu S, Akbulut N. The results of septal button insertion in the management of nasal septal perforation. J Laryngol Otol 1999;113(9):823-4.

16. Mullace M, Gorini E, Sbrocca M. Management of nasal septal perforation using silicone nasal septal button. Acta Otorhinolaryngol Ital 2006;26(4):216-8.

17. Combalia A, Carrera C. Squamous cell carcinoma: an update on diagnosis and treatment. Dermatol Pract Concept 2020;10(3):e2020066.

18. Altun S, Bal A, Arpaci E. Reconstruction of small and medium-sized nasal defects using an omega advancement flap. J Craniofac Surg 202;31(3):772-4.

19. Sapthavee A, Munaretto N, Toriumi DM. Skin grafts vs local flaps for reconstruction of nasal defects: a retrospective cohort study. JAMA Facial Plast Surg 2015;17(4):270-3

20. Bosch A, Vallecillo L, Frias Z. Cancer of the nasal cavity. Cancer 1976;37(3):145863.

21. Chang ET, Liu Z, Hildesheim A et al. Active and passive smoking and risk of nasopharyngeal carcinoma: A population-based case-control study in southern China. Am J Epidemiol 2017;185(12):1272-80. 